Published online: 17 August 2020

https://doi.org/10.1038/s41591-020-1047-5

References

1. Pallangyo, E., Nakate, M. G., Maina, R. \& Fleming, V. Midwifery 89, 102775 (2020).

2. Chatterji, P. \& Li, Y. National Bureau of Economic Research. http://www.nber.org/papers/w27173.pdf (2020).

3. Sherrard-Smith, E. et al. Nat. Med. https://doi.org/10.1038/ s41591-020-1025-y (2020).

4. Ranasinghe, S. et al. J. Ethnopharmacol. 166, 200-204 (2015).

5. Kapepula, P.M. et al. Am. J. Trop. Med. Hyg. https://doi.

org/10.4269/ajtmh.20-0820 (2020).
6. World Health Organization. https://www.who.int/news-room/ detail/23-04-2020-who-urges-countries-to-move-quickly-to-savelives-from-malaria-in-sub-saharan-africa (2020).

7. Dittrich, S. et al. Lancet Glob. Health 8, e879-e880 (2020).

8. World Health Organization. https://www.who.int/publications/m/ item/tailoring-malaria-interventions-in-the-covid-19-response (2020).

9. Alliance for Malaria Prevention. https://

allianceformalariaprevention.com/wp-content/uploads/2020/ 07/Key-guidance-for-ITN-distribution-during-COVIDtransmission-July-2020.pdf (2020).

10. World Health Organization. https://www.who.int/publications/ i/item/community-based-health-care-including-outreach-andcampaigns-in-the-context-of-the-covid-19-pandemic (2020).
Acknowledgements

R.A. and A.Z. are members of the Pan-African Network on Emerging and Re-emerging Infections (PANDORA-ID-NET) funded by the European and Developing Countries Clinical Trials Partnership the EU Horizon 2020 Framework Programme for Research and Innovation. A.Z. is a UK National Institute for Health Research senior investigator.

Competing interests

The authors declare no competing interests.

\title{
Vaccines targeting SARS-CoV-2 tested in
} humans

\author{
Vaccines directed against SARS-CoV-2 have been administered to healthy volunteers and have been shown to \\ stimulate a brisk humoral and cellular immune response. All vaccines were generally well tolerated with mostly \\ mild to moderate local and systemic reactions.
}

\section{Kathryn M. Edwards}

W ith the escalating numbers of global cases and fatalities associated with the newly discovered coronavirus SARS-CoV-2 (severe acute respiratory syndrome coronavirus 2 ), the recent publication of the results of three phase 1 vaccine trials is welcome news ${ }^{1-3}$. An article by Jackson et al. in the New England Journal of Medicine reports that within only 2 months after the viral sequence of SARS-CoV-2 was published, the first trial participant was given an mRNA vaccine that programmed the synthesis of a pre-fusion spike protein and triggered a brisk immune response ${ }^{1}$. Another report published in Nature highlights the safety and immunogenicity of a different mRNA vaccine $^{2}$, and a recent publication in Lancet describes a third virus-vectored vaccine with reassuring safety and immunogenicity results as well ${ }^{3}$.

The rapid generation of each of these three vaccines was the result of groundwork provided by previous phase 1 studies in humans of vaccines against SARS-CoV-1 and Middle East respiratory syndrome $e^{4,5}$, prior experience with the mRNA and adenovirus-vectored platforms $s^{6,7}$ and the evolution of new vaccine technologies over the past decade in response to other emerging pathogens $s^{8}$.

Jackson et al. report on the safety and immunogenicity of the mRNA vaccine administered to 45 healthy volunteers 18-55 years of age ${ }^{1}$. Two doses of vaccine were administered 1 month apart at three different dose levels, and the responses of the participants to these different regimes were compared. The experimental vaccine was associated with mild to moderate local and systemic adverse events, including local pain and tenderness at the injection site and headache, fever and malaise, with more-severe reactions noted at the highest vaccine dose. Reactions were more common after the second dose than after the first. However, the local and systemic reactions generally were not associated with an inability to continue the functions of everyday life and were self-limited. The authors found that the immune responses raised by participants after the second vaccine dose neutralized SARS-CoV-2 and generated $\mathrm{T}_{\mathrm{H}} 1$ cellular responses, the immune signatures projected to be associated with protection ${ }^{1}$. Since the immune responses to the middle dose and highest dose of vaccine were similar to each other and were greater than responses to the lowest dose, and since the reactogenicity of the highest dose was the greatest, the ongoing efficacy study is using the middle dose.

In the trial published in Nature, a lipid nanoparticle-formulated $\mathrm{mRNA}$ vaccine that encodes the trimerized SARS-CoV-2 spike glycoprotein receptor-binding domain was administered to 45 healthy adults 18-55 years of age who were randomly assigned to receive one of three different vaccine dosages or a placebo and were given two doses of vaccine (or placebo) 21 days apart ${ }^{2}$. Local reactions and systemic events were dose dependent and were generally mild to moderate and self-limited. Antibody concentrations were dose dependent, were boosted by the second vaccine dose and generated functional neutralizing antibody that exceeded levels seen in a panel of COVID-19 convalescent human serum. The efficacy phase of the trial has also begun with an mRNA vaccine that encodes the SARS-CoV-2 full-length spike glycoprotein.

In the phase 1 study in The Lancet, 1,077 participants were randomly assigned to receive either a chimpanzee adenovirus-vectored vaccine expressing the SARS-CoV-2 spike protein or a meningococcal quadrivalent conjugate vaccine $^{3}$. Ten of the participants received a second dose of vaccine. Local and systemic reactions were more common after receipt of the chimpanzee adenovirus spike protein vaccine. Systemic reactions included fever, chills, headache and malaise. Robust humoral and cellular immune responses were seen, and after an additional booster dose, all participants had neutralizing 


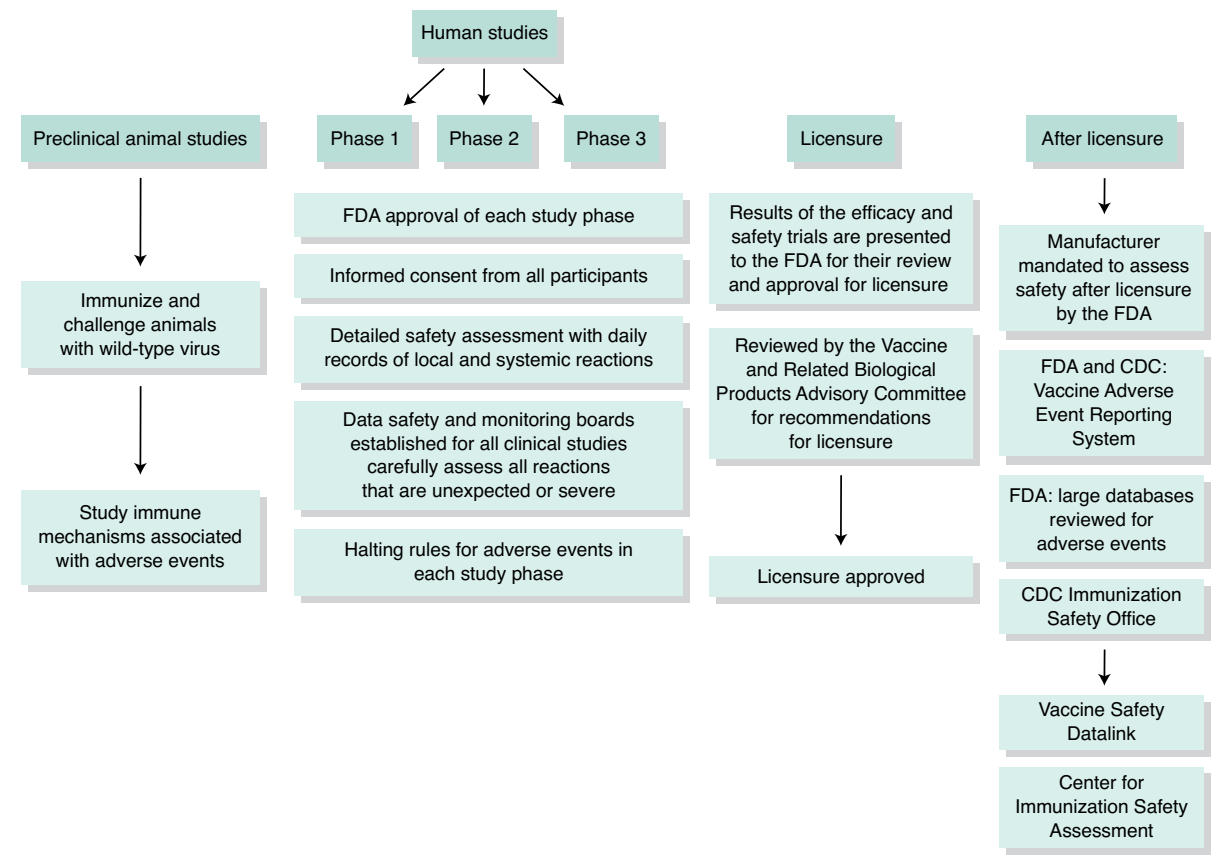

Fig. 1 | The processes required for the development of a vaccine against COVID-19 in humans. CDC, US Centers for Disease Control and Prevention.

antibody. Efficacy studies are also ongoing with this product.

Earlier animal studies with some vaccines against SARS-CoV-1 and Middle East respiratory syndrome, particularly inactivated whole virus vaccines, demonstrated that when vaccinated animals were exposed to wild-type virus, non-neutralizing antibody and $\mathrm{T}_{\mathrm{H}} 2$ cellular responses occurred and were associated with the pathologic infiltration of eosinophils into the lungs ${ }^{9}$, also called 'vaccine-enhanced disease.' The results of these three vaccine trials ${ }^{1-3}$ provide reassurance that the character of the immune response differs from what was seen with vaccine-enhanced disease in animals and features neutralizing antibody and $\mathrm{a} \mathrm{T}_{\mathrm{H}} 1$ cellular response ${ }^{10}$. Encouragingly, similar immune responses have been demonstrated in nonhuman primates immunized with vaccines against SARS-CoV-2 and challenged with wild-type virus ${ }^{11,12}$.

Given these encouraging early data, what is needed to ultimately immunize the global population of nearly 8 billion people with potentially two doses of vaccine (Fig. 1)? These three vaccines ${ }^{1-3}$ are currently being studied in phase 3 efficacy studies in which large, high-risk populations are being randomly assigned to receive either the vaccine or a placebo and are being monitored for the occurrence of laboratory-confirmed infection with SARS-CoV-2. According to a recently published guidance document from the
US Food and Drug Administration (FDA), a vaccine efficacy of at least $50 \%$ will required before a vaccine will be licensed for general use ${ }^{13}$. Also, before vaccine licensure in the USA, the safety and effectiveness of the vaccine will be promptly reviewed by the Vaccine and Related Biologics Products Advisory Committee, an independent committee of experts who will weigh in on all vaccine decisions. However, the ultimate decision will rest with the FDA. These processes are well established and are used for the licensure of all vaccines. In this case, the process will be expedited; the FDA has repeatedly shown its responsiveness during the pandemic.

Large government contracts have been issued for the construction of manufacturing facilities for these vaccines while they are still in the early phases of development, before the demonstration that they will be efficacious. This approach will support the rapid production of effective vaccines. Vaccines that use other technologies, including DNA vaccines, protein-based vaccines and inactivated whole virus vaccines, are being studied and, if promising, will also be rapidly evaluated in large-scale efficacy trials. It is also hoped that these studies will establish a serological correlate of protection, or a level of antibody that is associated with prevention of the disease, so that subsequent vaccines might be licensed on the basis of their attaining or surpassing that serological benchmark, which would potentially circumvent the need for additional efficacy trials. However, vaccines that attain these benchmarks would need to be shown to be safe and consistently immunogenic in large and varied population groups.

Vaccine prioritization after licensure will be the role of the Advisory Committee on Immunization Practices ${ }^{14}$. In addition, at the request of the US National Institutes of Health and the US Centers for Disease Control and Prevention, the National Academies of Sciences, Engineering, and Medicine have formed a committee that will develop an overarching framework to assist policymakers in the USA and global health communities in planning for equitable allocation of vaccines ${ }^{15}$.

Will these and other vaccines against SARS-CoV- 2 be accepted by the population? Over the past decade, there has been an increase in vaccine hesitancy. The origins of such hesitancy can generally be grouped into three broad areas: lack of concern about the disease or its consequences; concern for vaccine safety; and resistance to limitations of freedom imposed by mandatory vaccines. Achieving herd immunity requires that much of the population be immune. For a large proportion of the population to accept the new vaccine, all three areas of concern must be addressed. People will need to be convinced that SARS-CoV-2 poses a risk to them or their families, they will need to be reassured that the vaccine is safe and will not be associated with severe side effects, and they will need to believe that they should be vaccinated for the common good of the community. It is fair to say that current behaviors suggest that addressing all three issues will be challenging.

Some have questioned whether the rapid pace of vaccine development will short-circuit careful evaluation of vaccine safety. The public needs to know that vaccine safety is being meticulously addressed (Fig. 1). All clinical vaccine trials are approved by institutional review boards and rigidly adhere to the FDA guidelines. At every step of the clinical evaluation process, data safety and monitoring committees, independent of the vaccine manufacturers and investigators, and composed of experienced clinicians, epidemiologists and ethicists, review the safety data that are generated. Unusual or unexpected adverse events are meticulously evaluated and their rates are compared with the background rates of the occurrence of such events. All recipients of vaccine or placebo in the clinical trials will be monitored for adverse health outcomes for at least 1 year after vaccination and, for some, up to 3 years.

Vaccine scientists have met and reviewed the literature on adverse events associated 
with earlier vaccines against coronavirus and have proposed immunological conditions that should be met by candidate vaccines ${ }^{10}$. The Brighton Collaboration, a consortium of vaccine-safety professionals, has compiled a list of adverse consequences of COVID-19 disease to aid in characterizing potential vaccine-enhanced reactions ${ }^{16}$. This group is also formulating standard case definitions for global use to assess potential reactions temporally associated with vaccines. The existing vaccine-safety infrastructure of the FDA and the US Centers for Disease Control and Prevention for post-licensure vaccine monitoring is being enhanced to allow careful follow-up of vaccine recipients for any unexplained adverse events. Vaccine manufacturers will also be mandated to conduct post-licensure safety studies. These safety studies will be comprehensive and carefully monitored. The commitment to vaccine safety is, and should be, paramount.

\section{Kathryn M. Edwards (iD)}

Vanderbilt Vaccine Research Program, Vanderbilt University Medical Center, Nashville, TN, USA.

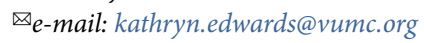

Published online: 24 August 2020

https://doi.org/10.1038/s41591-020-1048-4

\section{References}

1. Jackson, L.A. et al. N Engl. J. Med. https://doi.org/10.1056/ NEJMoa2022483 (2020).

2. Mulligan, M.J. et al. Nature https://doi.org/10.1038/s41586-0202639-4 (2020).

3. Folegatti, P.M. et al. Lancet https://doi.org/10.1016/S01406736(20)31604-4 (2020).

4. Martin, J. E. et al. Vaccine 26, 6338-6343 (2008).

5. Modjarrad, K. et al. Lancet 19, 1013-1022 (2019).

6. Pardi, N., Hogan, M. J., Porter, F. W. \& Weissman, D. Nat. Rev. Drug Discov. 17, 261-279 (2018).

7. Ewer, K. et al. Hum. Vaccin. Immunother. 13, 3020-3032 (2017).

8. Graham, B. S. \& Sullivan, N. J. Nat. Immunol. 19, 20-28 (2018).
9. Tseng, C.-T. et al. PLoS ONE 7, e35421 (2012).

10. Lambert, P. H. et al. Vaccine 38, 4783-4791 (2020).

11. van Doremalen, N. et al. bioRxiv https://doi. org/10.1101/2020.05.13.093195 (2020).

12. Corbett, K.S. et al.. N. Engl. J. Med. https://doi.org/10.1056/ NEJMoa2024671 (2020).

13. US Food and Drug Administration. https://www.fda.gov/ regulatory-information/search-fda-guidance-documents/ development-and-licensure-vaccines-prevent-covid-19 (2020).

14. Lee, G.M., Bell, B.P. \& Romero, J.R. JAMA https://doi, org/10.1001/jama.2020.13167 (2020).

15. The National Academies of Sciences, Engineering, and Medicine. https://www.nationalacademies.org/news/ 2020/07/national-academies-launch-study-on-equitableallocation-of-a-covid-19-vaccine-first-meeting-july-24 (2020).

16. Brighton Collaboration. https://brightoncollaboration.us/newwgs-develop-cds-covid-aesi/ (2020).

\section{Competing interests}

K.M.E. receives grant funding from the CDC (related to COVID 19) and the NIH, is a consultant for BioNet and IBM, and is a member of data safety and monitoring committees for Pfizer (related to COVID 19), the NIH, Sanofi Pasteur, X-4 Pharma, Seqirus and Moderna. 